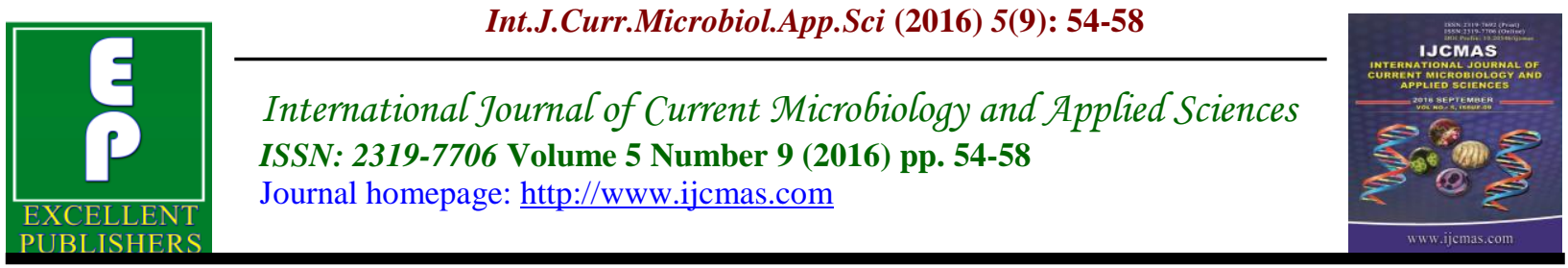

Original Research Article

http://dx.doi.org/10.20546/ijcmas.2016.509.007

\title{
Mycological Shift of Candida Species Isolated from Blood Culture of Pediatric Patients
}

\author{
J. Lavanya ${ }^{1}$, Suchitra Mishra ${ }^{2}$, Manoj Jais ${ }^{3}$ and Mita D. Wadekar ${ }^{1}$ \\ ${ }^{1}$ Subbaiah Institute of Medical Sciences, Shimoga, Karnataka State, India \\ ${ }^{2} \mathrm{Hi}-\mathrm{Tech}$ Medical College and hospital, Rourkela, India \\ ${ }^{3}$ Lady Hardinge Medical College, New Delhi, India
}

*Corresponding author

Keywords

Mycological shift, non albicans

candida,

Candida albicans, blood culture, pediatric ICU.

\section{Article Info}

Accepted:

07 August 2016

Available Online:

10 September 2016

\section{A B S T R A C T}

With emerging immunocompromised status, fungal infections are taking the leading role as the causative agents in most of the dreadful infections. Candidemia is becoming a leading cause of healthcare associated infections nowadays among pediatric population. A Retrospective observational study was done at a tertiary care hospital, New Delhi. 60 candida species from blood culture were analysed for speciation and prevalence in the hospital. Blood culture was performed by Bact/Alert 3D system [Biomerieux,France]. Chromogenic agar and Vitek 2 YST identification card (bioMerieux, France) was used for speciation. Blood culture results showed that Non albicans candida (73.3\%) species were the majority compared to candida albicans (26.6\%). Candida tropicalis $(33.3 \%)$ was the most common species isolated followed by C.glabrata (21.6\%). Majority of the candida species were isolated from pediatric ICU (53.3\%) compared to pediatric ward (46.6\%). This study shows the clinical significance and mycological shift of candida species in blood culture of pediatric population with predominance of non albicans candida.

\section{Introduction}

Health care associated infections are becoming a leading cause of morbidity and mortality among patients with economical burden on the developing countries like India. Nowadays fungal infections are becoming the leading cause of health care associated infections among children and among immunocompromised individuals.

Candida albicans has been the most common cause of bloodstream infections, but nowadays nonalbicans candida (NAC) is becoming predominant. Azoles bare the most commonly used antifungal agent to treat fungal infections; but NAC are resistant to azoles, which is a matter of concern (Gunjan Shrivastava et al., 2015).

Children are more susceptible to fungal infections because of risk factors like low birth weight, poor nutrition, admissions to ICU etc. most of the pathogenic species like 
C.albicans, C.glabrata, C.guiilermondii, C.parapsilosis, C.dubliniensis, C.krusei and others are the normal commensals of human body inhabiting GIT, urinary tract and skin. Whenever the immunity of the individual reduces they predominate and become pathogenic causing many complications.

Candida albicans has been the leading cause of blood stream infections but many studies have reported NAC to be overtaking C.albicans. This mycological shift is very significant as they differ in the susceptibility to antifungals (Oberoi et al., 2012; Goel et al., 2009).

Thus the present study has been carried out to determine the changing pattern of candida species and their prevalence in the different wards of the hospital.

\section{Material and Methods}

The present retrospective study was carried out in the department of Microbiology, lady hardinge medical college, New Delhi. A total of 200 peripheral venous blood samples from children suspected of septicemia were collected aseptically (District Laboratory practice in tropical countries part 2 Monica Cheesbrough, 2000) and prior to antibiotic therapy during the period from January 2014 to March 2014.

Blood culture was performed by automated Bact/Alert 3D system [Biomerieux, France]. The Bact/Alert $\mathrm{R}$ is a growth-based technology and works on the principle that as microorganisms grown, they produce $\mathrm{CO}$; these systems use colorimetric detection of $\mathrm{CO} 2$ production from the growth of organism. It is a qualitative procedure for enhanced recovery and detection of aerobic and facultative anaerobic microorganism (bacteria and fungi) blood. The culture bottles used in this system were aseptically inoculated with $2 \mathrm{ml}$ of venous blood. The blood culture bottles that were indicated as positive were subcultured onto Blood agar and MacConkey agar media. Candida species identification was based on cultural characteristics, Gram stain and assessment by Germ tube test and colony on corn meal agar. Speciation of all isolates was done by chromogenic media (HiChrome, Hi Media Mumbai, Pvt. Ltd) and Vitek 2 YST identification card (bioMerieux, France). Strict quality control was followed during the entire study period (Bailey and Scott's. Diagnostic Microbiology; Baron et al., 2005).

\section{Results and Discussion}

In our study non albicans candida (73.3\%) has outnumbered Candida albicans (26.6\%) significantly as shown in fig I. Only a few studies from India have reported candidaemia rates of 6-18 per cent and an increase in isolation of non-albicans Candida species from blood samples (Shivaprakasha et al., 2007; Chakrabarti et al., 2009).

In our study, Candida infections were more prevalent in ICU compared to pediatric ward as illustrated in Table I and fig II. This could be due to longer duration of hospital stay, antibiotics usage and immunocompromised states in ICU. Among the various risk factors implicated in the acquisition of candidemia or invasive candidiasis in neonates, the most important factor is low birth weight (LBW) and prematurity. In a study by Lee et al., neonates with birth weights less than $1250 \mathrm{~g}$ were found to be at a greater risk of getting candidemia or meningitis caused by Candida. Such neonates also had a higher chance of developing complications like intraventricular haemorrhage and greater mortality rate than control neonates. A few Indian studies have also evaluated the risk 
factors in neonates associated with candidemia and found that the isolation of Candida species from any other focus of infection and indwelling intravascular catheters to be the most common risk factors associated with this condition. Admission in the ICU is another important risk factor for candidemia in neonates (Blumberg et al.,
2001). Among the non-albicans Candida species, $C$. glabrata has emerged as an important opportunistic pathogen worldwide. It is the most common yeast isolated as part of normal flora and its role as a pathogen has only been recognized in the past few decades.

Table.1 Distribution of candida species among pediatric population

\begin{tabular}{|c|c|c|c|c|}
\hline Sl. No & Candida species & ICU & Pediatric ward & Total \\
\hline 1. & C. albicans & $9(28 \%)$ & $7(25 \%)$ & $\mathbf{1 6}(\mathbf{2 6 . 6 \%})$ \\
\hline 2. & C. tropicalis & $12(37.5 \%)$ & $8(28.5 \%)$ & $\mathbf{2 0}(\mathbf{3 3 . 3} \%)$ \\
\hline 3. & C.glabrata & $7(21 \%)$ & $6(21 \%)$ & $\mathbf{1 3}(\mathbf{2 1 . 6 \%})$ \\
\hline 4. & C.krusei & $3(9 \%)$ & $4(14 \%)$ & $\mathbf{7 ( 1 1 . 6 \% )}$ \\
\hline 5. & C. parapsilosis & $1(3 \%)$ & $3(10 \%)$ & $\mathbf{4}(\mathbf{6 . 6 \%})$ \\
\hline & Total & $\mathbf{3 2}(\mathbf{5 3 . 3 \%})$ & $\mathbf{2 8}(\mathbf{4 6 . 6 \%})$ & $\mathbf{6 0}$ \\
\hline
\end{tabular}

Fig.1 Prevalence of candida species in pediatric ICU and pediatric ward

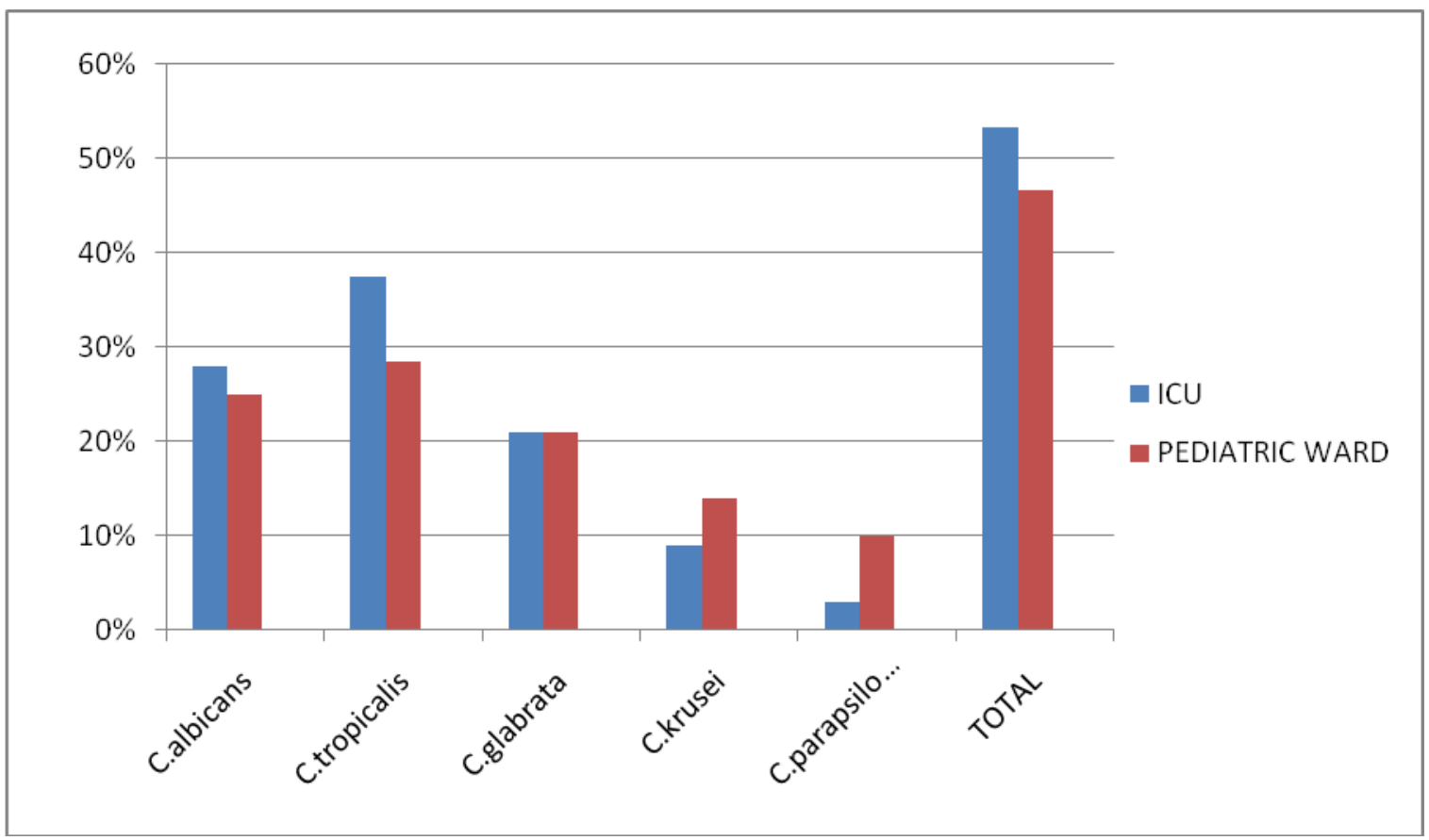


Fig.2 Prevalence of C. albicans and Non albicans Candida

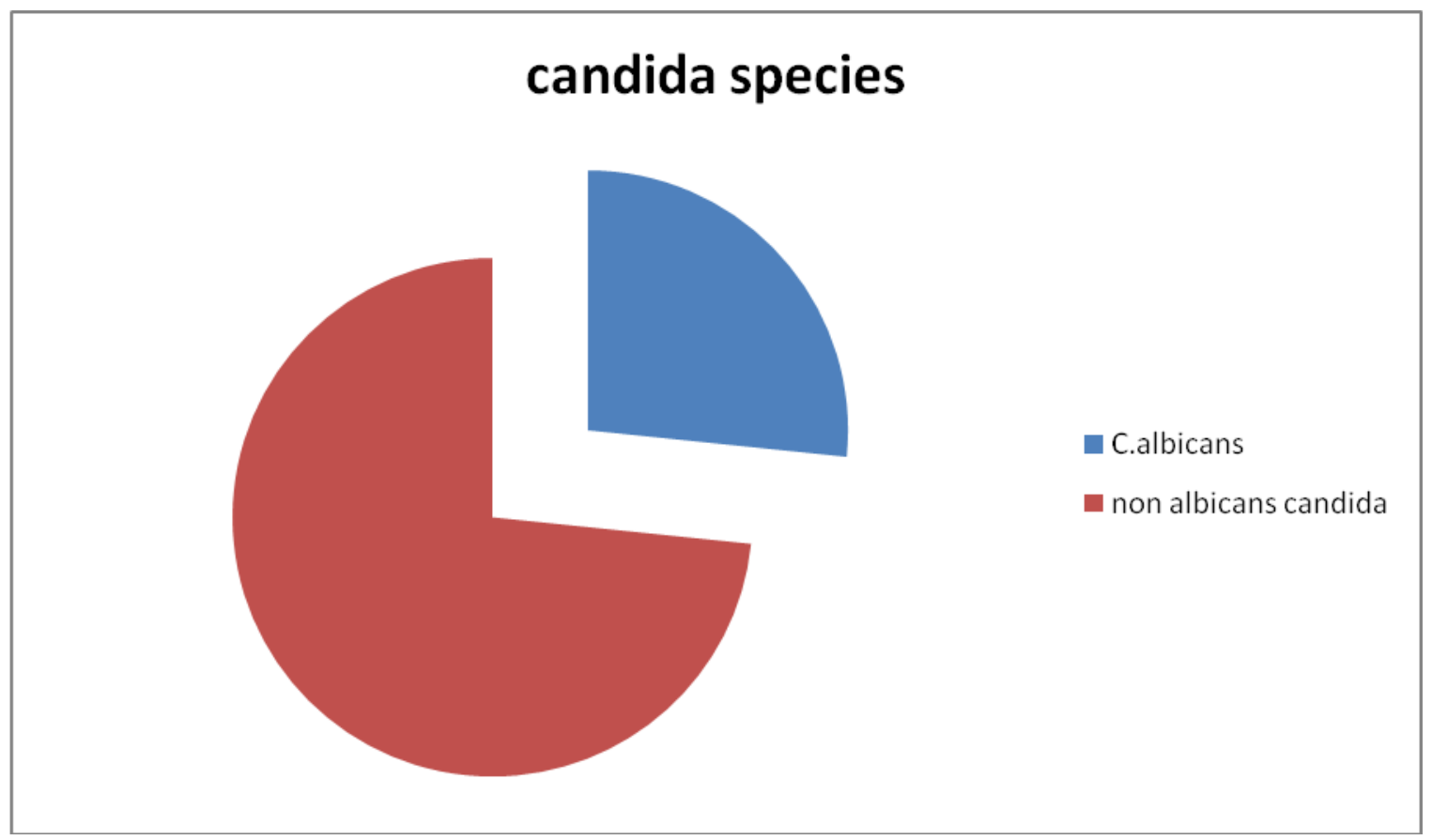

Trick et al. reported a considerable increase in the isolation rate of $C$. glabrata from BSI in U.S ICUs which is in correlation with our study (Trick et al., 2002; Malani et al., 2005). Although the risk factors for candidemia due to $C$. glabrata appear to be the same as for candidemia in general, this particular species has emerged prominently among patients with haematological malignancies.

In conclusion, there has been a rise in the occurrence of candidaemia cases in our tertiary care hospital over the last decade. A significant epidemiological shift to higher isolation of non-albicans Candida species was noticed. A study conducted at a tertiary hospital in New Delhi also concluded similarly and found out that the high usage of fluconazole appeared to have played a role in this shift. However, it may be recognised that other events like patient specific risk factors might have also contributed in selection of different species.
Decreased susceptibility to fluconazole with the threat of emergence of cross-resistance to voriconazole in the background of high azole consumption may limit the use of these agents as empirical therapy for Candida BSI which is a matter of concern.

\section{References}

Bailey and Scott's. Diagnostic Microbiology. In: Betty A, Forbes Daniel, Sahmalice S, editors. 11 th ed. Weissfelp.

Baron E.J. et al. 2005. Cumitech 1C Blood cultures iv. Coordinating. In: Baron EJ, editor. Washington: ASM Press.

Blumberg, H.M., Jarvis, W.R., Soucie, J.M., Edwards, J.E., Patterson, J.E., Pfaller, M.A. 2001. Risk factors for candidal bloodstream infections in surgical intensive care unit patients: The NEMIS Prospective Multicenter Study. Clin. Infect. Dis., 33: 177-86. 
Chakrabarti, A., Chatterjee, S.S., Rao, K.L., Zameer, M.M., Shivaprakash, M.R., Singhi, S., et al. 2009. Recent experience with fungaemia: change in species distribution and azole resistance. Scand J. Infect. Dis., 41: 275-84.

District Laboratory practice in tropical countries part 2 Monica Cheesbrough; 2000.

Goel, N., Ranjan, P.K., Agarwal, R., Chaudhary, U., Sanjeev, N. 2009. Emergence of non-albicans Candida in septicaemia and antifungal susceptibility: Experience from a tertiary care centre. J. Lab. Physicians, 1: 53-5.

Gunjan Shrivastava, et al. 2015. Neonatal Candidemia: Clinical Importance of Species Identification. Sifa Med. J., Volume : 2, Issue : 2, Page : 37— 40 .

Gupta, N., Mittal, N., Sood, P., Kumar, S., Kaur, R., Mathur, M.D. 2001. Candidemia in neonatal intensive care Unit. Indian J. Pathol. Microbiol., 44: 45-8.

Lee, B.E., Cheung, P.Y., Robinson, J.L,. Evanochko, C., Robertson, C.M. 1998.
Comparative study of mortality and morbidity in premature infants (Birth Weight, 1,250 g) with Candidemia or Candidal Meningitis. Clin. Infect. Dis., 27: 559-65.

Malani, A,. Hmoud, J., Chiu, L., Carver, P.L., Bielaczyc, A., Kauffman, C.A. 2005. Candida glabrata fungemia: Experience in a tertiary care center. Clin. Infect. Dis., 41(9): 75-81.

Oberoi, J.K., Wattal, C. et al. 2012. Nonalbicans Candida species in blood stream infections in a tertiary care hospital at New Delhi, India. Indian J. Med. Res., 136: 997-1003.

Shivaprakasha, S., Radhakrishnan, K., Karim, P.M. 2007. Candida spp. other than Candida albicans: a major cause of fungaemia in a tertiary care centre. Indian J. Med. Microbiol., 25: 405-7.

Trick, W.E., Fridkin, S.K., Edwards, J.R., Hajjeh， R.A., Gaynes， R.P. 2002. Secular trend of hospital-acquired candidemia among intensive care unit patients in the United States during 1989-1999. Clin. Infect. Dis., 35: 62730.

\section{How to cite this article:}

Lavanya, J., Suchitra Mishra, Manoj Jais and Mita D. Wadekar. 2016. Mycological Shift of Candida Species Isolated from Blood Culture of Pediatric Patients. Int.J.Curr.Microbiol.App.Sci. 5(9): 54-58. doi: http://dx.doi.org/10.20546/ijcmas.2016.509.007 